\title{
Síndrome coronario agudo en edad temprana. Descripción de una cohorte ¿Se está estratificando adecuadamente el riesgo?
}

\author{
Acute coronary syndrome at early age. Description of a cohort. Is the risk being properly \\ stratified?
}

\author{
Mateo Mejía-Zuluaga ${ }^{1 *}$, Orlando Castaño-Cifuentes ${ }^{1}$, Alejandro Arroyave-Carvaja², \\ Mauricio Duque-Ramírez ${ }^{3}$, Elsa M. Vásquez-Trespalacios ${ }^{4}$ y Viviana Quintero-Yepes ${ }^{2}$ \\ ${ }^{1}$ Cardiología Clínica, Universidad CES; ${ }^{2}$ Servicio de Cardiología, Clínica El Rosario; ${ }^{3}$ Departamento de Cardiología y Electrofisiología, Universidad \\ CES; ${ }^{4}$ Servicio de Epidemiología, Universidad CES. Medellín, Colombia
}

\section{Resumen}

Objetivo: Describir las características demográficas, ecocardiográficas y angiográficas, así como la presentación clínica, los hábitos, los antecedentes y los desenlaces de pacientes menores de 50 años atendidos por síndrome coronario agudo (SCA). Método: Estudio observacional, retrospectivo y descriptivo, con análisis univariado y bivariado, que incluyó información de pacientes atendidos en una clínica de tercer nivel de la ciudad de Medellín, Colombia. Resultados y conclusiones: Se identificaron 127 casos, de los que el 79.5\% eran hombres y el tipo de SCA más frecuente fue el infarto agudo de miocardio (IAM) no ST (43\%), seguido por el IAM ST (23\%). Los factores de riesgo más prevalentes fueron el sobrepeso, la obesidad y las alteraciones del perfil lipídico (triglicéridos elevados y colesterol ligado a lipoproteínas de alta densidad bajo). Se encontró baja prevalencia en tabaquismo y diabetes mellitus. El 78\% de los casos tenían una fracción de eyección del ventrículo izquierdo preservada, con presencia de trastornos segmentarios en la ecocardiografía basal en un 55\%. El 20\% tenían enfermedad coronaria grave de tres o más vasos. La coronaria más comprometida fue la arteria descendente anterior (58.2\%). Las escalas de riesgo GRACE, Killip-Kimball y TIMI clasificaron a los pacientes en bajo riesgo, siendo congruentes con los pacientes estudiados intrahospitalariamente, pues se encontró una mortalidad del 0\%; sin embargo, debe primar el criterio clínico al tomar decisiones sobre el método de estratificación y el ámbito hospitalario o ambulatorio. Se plantea la necesidad de establecer protocolos para una evaluación funcional y estructural de la microcirculación coronaria en pacientes con cuadro clínico sugestivo, electrocardiograma y pruebas de inducción de isquemia positivas a pesar de tener coronariografía sin lesiones epicárdicas.

Palabras clave: Enfermedad coronaria. Enfermedad cardiovascular. Síndrome coronario agudo. Edad. Jóvenes.

\section{Abstract}

Objective: To describe the demographic, echocardiographic, angiographic characteristics, clinical presentation, habits, history, and outcomes of patients younger than 50 years attended for acute coronary syndrome (ACS). Method: An observational, retrospective and descriptive study was performed, with univariate and bivariate analysis that included information of patients treated at a third level clinic in Medellín, Colombia. Results and conclusions: 127 cases were identified where $79.5 \%$ were men and the most frequent type of ACS was NSTEMI (43\%) followed by STEMI (23\%). The most prevalent risk factors were

\section{Correspondencia:}

*Mateo Mejía-Zuluaga

E-mail: mmzuluaga@ hotmail.com
Fecha de recepción: 18-06-2020

Fecha de aceptación: 15-09-2020

DOI: 10.24875/RCCAR.M22000117
Disponible en internet: 22-02-2022 Rev Colomb Cardiol. 2022;29(1):49-56 www.rccardiologia.com 0120-5633 / @ 2022 Sociedad Colombiana de Cardiología y Cirugía Cardiovascular. Publicado por Permanyer. Este es un artículo open access bajo la licencia CC BY-NC-ND (http://creativecommons.org/licenses/by-nc-nd/4.0/). 
overweight, obesity, and changes in the lipid profile (high triglycerides and low HDL), with low prevalence in smoking and diabetes mellitus. $78 \%$ of the cases had preserved LVEF with the presence of segmental contractility disorders on baseline echocardiography in $55 \%$ of the patients. $20 \%$ had severe coronary heart with 3 or more vessel disease. The most frequently compromised coronary artery was the LAD (58.2\%). GRACE, Killip-Kimball and TIMI risk scales classified the patients as low risk, being congruent with the hospitalized patients with a mortality rate of $0 \%$; However, clinical criteria should prevail when making decisions about the stratification method and the place (in-hospital or outpatient setting). The need to establish protocols for a functional and structural evaluation of coronary artery microcirculation in patients with a suggestive clinical history, ECG, and positive ischemia induction tests, despite having coronary angiography without epicardial lesions, is demanded.

Keywords: Coronary disease. Cardiovascular disease. Acute coronary syndrome. Age. Young.

\section{Introducción}

La enfermedad coronaria es la primera causa de muerte en todo el mundo, incluso por encima de las enfermedades oncológicas que vienen aumentando su incidencia anual paulatinamente; sin embargo, es poco probable que vaya a ser desplazada a corto plazo, pues es una enfermedad cuya prevalencia aumenta a medida que la población envejece ${ }^{1-3}$. Pese a implementar estrategias para el control temprano de los factores de riesgo tradicionales, la incidencia de eventos coronarios en la población joven continúa siendo un gran problema de salud pública, ya que conlleva morbilidad y mortalidad asociadas, y una fisiopatología parcialmente comprendida, lo que dificulta establecer medidas de prevención efectivas ${ }^{4-6}$, empezando por la definición de adulto joven, pues hasta ahora no existe un consenso general de expertos ni una recomendación oficial al respecto.

Algunos autores consideran el punto de corte de edad de 40 años y otros proponen 45 años o incluso 55 años, dado que es a partir de esta edad cuando los factores de riesgo cardiovasculares tienen más impacto en la salud del adulto ${ }^{7-15}$. El estudio Framingham definió el infarto agudo de miocardio (IAM) en paciente joven con una edad menor de 55 años ${ }^{16}$; no obstante, una de las definiciones más reportadas para establecer enfermedad coronaria temprana o en paciente joven es hombres y mujeres con edad $\leq 50$ años ${ }^{17-19}$.

Se han realizado estudios retrospectivos en otras regiones de Colombia ${ }^{20}$, los cuales han mostrado que los principales factores de riesgo son el tabaquismo, la hipercolesterolemia familiar, la diabetes mellitus, la hipertensión arterial, el consumo de sustancias psicoactivas y las trombofilias ${ }^{5,21}$. A la fecha, no se han realizado descripciones de la población que presenta enfermedad coronaria temprana en la región noroeste del país, con la cual se podrían demostrar similitudes o diferencias en cuanto a las características sociodemográficas y a la prevalencia de factores de riesgo cardiovasculares. La identificación temprana, el mejor control de factores de riesgo diferentes a los tradicionales y la decisión de realizar estudios intrahospitalarios o ambulatorios tomados con un mayor conocimiento del tema, permitirían detectar factores de riesgo de forma temprana y posibles etiologías, para así impactar de manera favorable los diferentes desenlaces de la enfermedad cardiovascular a corto y largo plazo en la población joven.

El objetivo de este estudio fue describir las características demográficas, ecocardiográficas y angiográficas, así como la presentación clínica, los hábitos, los antecedentes y los desenlaces de pacientes menores de 50 años atendidos por síndrome coronario agudo (SCA) en una institución de tercer nivel de la ciudad de Medellín, Colombia.

\section{Método}

Se llevó a cabo un estudio observacional, retrospectivo y descriptivo, con análisis univariado y bivariado, que incluyó información de pacientes atendidos en una clínica de tercer nivel de la ciudad de Medellín, Colombia, durante el período comprendido desde el 1 de enero de 2016 hasta el 30 de junio de 2019, con diagnóstico de SCA según los criterios internacionales de la cuarta definición de IAM (IAM con elevación del segmento ST, IAM sin elevación del segmento ST y angina inestable) ${ }^{3}$.

Se incluyeron pacientes con edad entre 18 y 50 años, atendidos con diagnóstico de enfermedad coronaria presentándose como un SCA. Se excluyeron pacientes con datos incompletos en la historia clínica electrónica, definidos como ausencia de más del 50\% de las variables, dolor torácico de origen no cardíaco o miocarditis aguda.

Se seleccionó la información a través de fuente secundaria, revisando las historias clínicas realizadas en urgencias y hospitalización, según los códigos diagnósticos CIE-10 para SCA y enfermedad coronaria 
(I200, I201, I208, I209, I210, I211, I212, I213, I214, I219, I220, I221, I228, I229, I248, I249, I250, I255, I256, I258 e 1259). Se revisaron un total de 286 historias clínicas, de las cuales 120 cumplían los criterios de exclusión debido principalmente a falta de datos $(<50 \%)$, puesto que se definía la realización de estudios ambulatoriamente por parte del médico, seguido por etiología no cardíaca. De las 166 historias que no cumplían los criterios de exclusión, se determinó excluir adicionalmente aquellos pacientes con angina inestable en quienes no se logró documentar isquemia por métodos no invasivos de estratificación coronaria o por arteriografía coronaria (Fig. 1). En total, ingresaron 127 pacientes al análisis estadístico. Los datos se analizaron en el paquete estadístico SPSS versión 21. Para los datos descriptivos se obtuvieron promedios y medianas con sus respectivas medidas de dispersión. Las variables cualitativas se presentan con frecuencias absolutas y relativas.

Se documentaron variables relacionadas con la presentación clínica (tipo de dolor, irradiación, disnea o síncope), antecedentes personales y familiares del paciente, estratificación del riesgo de pacientes con IAM definido como se establece en las guías europeas para el manejo de estos pacientes ${ }^{2,22}$, hallazgos ecocardiográficos sobre la función de ambos ventrículos cuyas definiciones usadas son las recomendadas por las guías de la American Society of Echocardiography ${ }^{23}$, hallazgos en la angiografía coronaria, incluyendo vaso comprometido y número de vasos; y resultados de exámenes de laboratorio que incluían perfil lipídico, hemoglobina glucosilada $(\mathrm{HbA} 1 \mathrm{c})$ y función renal, entre otros que ayudaran a estadificar el riesgo cardiovascular del paciente.

Desde el punto de vista ético, este es un estudio sin riesgo, en el cual se aplican los principios éticos de autonomía y beneficencia y no maleficencia, respetando la intimidad y la confidencialidad de la información. Los investigadores contaron con consentimiento informado del representante legal de la institución investigadora, la Universidad CES, y de la institución donde se desarrolló la investigación, Clínica El Rosario, sede Tesoro.

\section{Resultados}

En la tabla 1 se muestran las características clínicas y sociodemográficas de la población evaluada.

El sexo masculino fue el más frecuente, con un $79.5 \%$. El tipo de SCA más presentado por los pacientes fue el IAM no ST (43\%), seguido por el IAM ST y

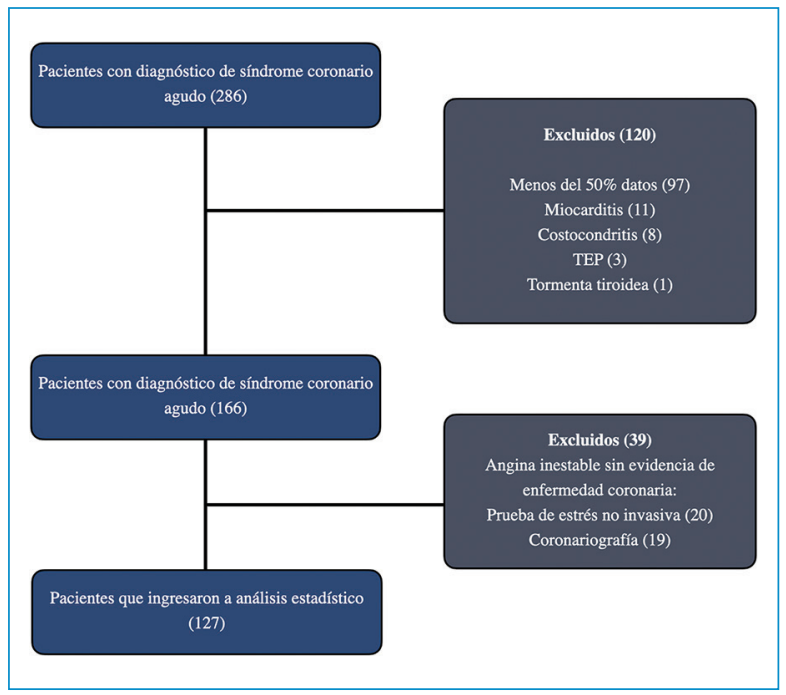

Figura 1. Diagrama de flujo de selección y exclusión de pacientes. TEP: tromboembolia pulmonar.

la angina inestable, ambos con un $23 \%$. Las diferentes escalas de valoración de riesgo y pronóstico utilizadas (Killip-Kimball, TIMI y GRACE), en su mayoría clasificaban a los pacientes como de bajo riesgo (edad como una variable de gran peso), correlacionándose con una baja mortalidad intrahospitalaria.

Todos los pacientes fueron hospitalizados en la unidad de cuidados coronarios, 17 requirieron estancia en la unidad de cuidados intensivos (UCI) y, de esos, nueve debido a que se les realizó cirugía de revascularización miocárdica (CABG, coronary artery bypass grafting), por lo que entraron a la $\mathrm{UCl}$ como protocolo durante su posoperatorio inmediato y no por deterioro hemodinámico. Se tuvo un promedio de días de hospitalización de 4.13 días (mínimo 1 día y máximo 44 días).

En cuanto a la presentación clínica, el dolor torácico opresivo y retroesternal fue el más reportado, en un $85 \%$ de los casos, seguido por el dolor de tipo punzante $(8.6 \%)$ y con menos frecuencia urente $(2.36 \%)$; de estos, el $48.8 \%$ presentaban irradiación del dolor, en cuyo caso el miembro superior izquierdo y la mandíbula o el mentón fueron los sitios más referidos. El síncope no es una forma frecuente de presentación; sin embargo, el $4.72 \%$ de los pacientes lo presentaron.

Los antecedentes personales se encontraron con la siguiente distribución: hipertensión arterial 44\%, dislipidemia 44\%, tabaquismo activo 17\%, antecedente familiar de enfermedad coronaria $22 \%$, enfermedad coronaria previa o equivalente a ateroesclerosis $15 \%$, y diabetes mellitus $11 \%$. De los 56 pacientes con 
Tabla 1. Características clínicas y sociodemográficas de los pacientes

\begin{tabular}{|c|c|c|}
\hline & $\begin{array}{c}\text { Todos los } \\
\text { pacientes }(n=127)\end{array}$ & $\%$ \\
\hline Sexo masculino & 101 & 79.5 \\
\hline Tipo de SCA & - & - \\
\hline IAM ST & 36 & 28.34 \\
\hline IAM no ST & 55 & 43.30 \\
\hline Angina inestable & 36 & 28.34 \\
\hline Killip-Kimball & & \\
\hline 1 & 117 & 92.12 \\
\hline II & 6 & 4.72 \\
\hline III & 0 & 0 \\
\hline IV & 4 & 3.14 \\
\hline GRACE & & \\
\hline Riesgo bajo & 124 & 97.63 \\
\hline TIMI & & \\
\hline$\leq 1$ & 38 & 29.92 \\
\hline 2 & 65 & 51.18 \\
\hline$\geq 3$ & 24 & 18.89 \\
\hline Mortalidad intrahospitalaria & 0 & 0 \\
\hline ICP & 107 & 84.25 \\
\hline CABG & 9 & 7.08 \\
\hline Trombólisis & 3 & 2.36 \\
\hline $\begin{array}{l}\text { Días de hospitalización } \\
\text { (promedio) }\end{array}$ & 4.13 & - \\
\hline Uso de terapia vasoactiva & 18 & 14.2 \\
\hline Uso de vasodilatadores & 37 & 29.1 \\
\hline Dolor torácico & - & - \\
\hline Opresivo & 109 & 85.82 \\
\hline Punzante & 11 & 8.66 \\
\hline Urente & 3 & 2.36 \\
\hline Irradiado & 62 & 48.81 \\
\hline Disnea & 54 & 42.51 \\
\hline Síncope & 6 & 4.72 \\
\hline Antecedentes personales & - & - \\
\hline Diabetes mellitus & 14 & 11.02 \\
\hline IMC & - & - \\
\hline Peso normal $\left(18-24.9 \mathrm{~kg} / \mathrm{m}^{2}\right)$ & 37 & 29.13 \\
\hline Sobrepeso $\left(25-29.9 \mathrm{~kg} / \mathrm{m}^{2}\right)$ & 65 & 51.18 \\
\hline Obesidad $\left(\geq 30 \mathrm{~kg} / \mathrm{m}^{2}\right)$ & 25 & 19.68 \\
\hline Dislipidemia & 56 & 44.09 \\
\hline Hipertensión arterial & 56 & 44.09 \\
\hline Tabaquismo activo & 22 & 17.32 \\
\hline ERC estadio I & 97 & 76.37 \\
\hline ERC estadio II & 24 & 18.89 \\
\hline ERC estadio III & 4 & 3.14 \\
\hline ERC estadio IV & 0 & 0 \\
\hline ERC estadio V & 2 & 1.57 \\
\hline Aterosclerosis previa & 19 & 14.96 \\
\hline $\begin{array}{l}\text { Antecedente familiar de } \\
\text { enfermedad coronaria }\end{array}$ & 28 & 22.04 \\
\hline
\end{tabular}

Tabla 1. Características clínicas y sociodemográficas de los pacientes (Continuación)

\begin{tabular}{|l|c|c|} 
& $\begin{array}{c}\text { Todos los } \\
\text { pacientes }(\mathbf{n}=\mathbf{1 2 7})\end{array}$ & $\%$ \\
\hline Ecocardiografía transtorácica & - & - \\
FEVI $\geq 50 \%$ & 99 & 77.95 \\
FEVI $\leq 40$ & 9 & 7.08 \\
\hline TAPSE $\geq 17$ & 100 & 78.74 \\
\hline Trastornos segmentarios de la & 77 & 55.11 \\
contractilidad miocárdica & & \\
\hline
\end{tabular}

CABG: coronary artery bypass grafting; ERC: enfermedad renal crónica; FEVI: fracción de eyección del ventrículo izquierdo; GRACE: Global Registry of Acute Coronary Events; IAM: infarto agudo de miocardio; ICP: intervención coronaria percutánea; IMC: índice de masa corporal; SCA: síndrome coronario agudo; TAPSE: tricuspid annular plane systolic excursion; TIMI: Thrombolysis in Myocardial Infarction.

hipertensión arterial, el 89\% tenía tratamiento con inhibidores de la enzima convertidora de angiotensina o antagonistas del receptor de la angiotensina II. Para el grupo con antecedente de dislipidemia, solo el $83 \%$ recibía tratamiento farmacológico, distribuido así: el $51 \%$ estatinas de alta intensidad, el $26.7 \%$ estatinas de moderada intensidad y el $5.3 \%$ estatinas de baja intensidad.

El $71 \%$ de los pacientes se encontraron con sobrepeso y obesidad evaluado por el índice de masa corporal (IMC). Las alteraciones en el perfil lipídico que predominaron fueron los triglicéridos elevados con el colesterol unido a lipoproteínas de alta densidad (HDL) bajo (triglicéridos $216 \mathrm{mg} / \mathrm{dl}$ en promedio, con un rango de $51-809 \mathrm{mg} / \mathrm{dl}$; HDL promedio de $35.5 \mathrm{mg} / \mathrm{dl}$, con un rango de $21-72 \mathrm{mg} / \mathrm{dl})$; colesterol unido a lipoproteínas de baja densidad (LDL) promedio $109 \mathrm{mg} / \mathrm{dl}$, con un rango de 30-259 mg/dl. Al evaluar los pacientes que tenían antecedente de enfermedad cardiovascular aterosclerótica $(n=18)$, cuatro se encontraban en metas de LDL, siete estaban fuera de metas y en los otros siete no se obtuvieron datos. Cabe anotar que 15 recibían estatinas de alta intensidad, uno de moderada intensidad, uno de baja intensidad y el último no tomaba medicamento hipolipidemiante.

En cuanto a la valoración de la alteración en el metabolismo de los hidratos de carbono, se encontró un promedio de glucosa capilar al azar de $118 \mathrm{mg} / \mathrm{dl}, 19$ pacientes (14.9\%) con HbA1c en rango de prediabetes y 13 pacientes (10.2\%) en rango de diabetes, y 7 (5.5\%) tenían una $\mathrm{HbA1c} \geq 8 \%$. Entre los pacientes diabéticos, el $9.44 \%(n=12)$ recibía tratamiento farmacológico con terapia hipoglucemiante (Tabla 2).

La ecocardiografía transtorácica basal demostró que el $78 \%$ de los pacientes tenían una fracción de 
eyección del ventrículo izquierdo preservada y una adecuada función del ventrículo derecho (TAPSE $\geq$ 17); se documentaron trastornos segmentarios de la contractilidad en el $55 \%$ de los casos.

En cuanto a los hallazgos de la angiografía coronaria (Fig. 2), el $43.3 \%$ de los pacientes tuvieron compromiso de un solo vaso, el $19.6 \%$ de dos vasos y el $20.47 \%$ de tres o más vasos coronarios; solo el $1.6 \%$ presentó compromiso de tronco principal izquierdo o equivalente. Sin embargo, el $16.5 \%$ de los pacientes tenían coronarias sin lesiones ateroscleróticas significativas; de este último grupo, el $5 \%$ debutó con IAM con elevación del segmento ST, el $45 \%$ con IAM sin elevación del segmento ST y el $50 \%$ con angina inestable. No se realizó medición de reserva de flujo fraccional (FFR), índice instantáneo sin ondas (iFR), test de provocación de vasoespasmo ni evaluación fisiológica de la microcirculación.

El principal vaso afectado fue la arteria descendente anterior, comprometida en 74 pacientes (58.26\%), seguida por la coronaria derecha en 52 pacientes $(50.9 \%)$ y, por último, la circunfleja en 44 pacientes (34.64\%). En el subgrupo de ausencia de lesiones ateroscleróticas, los hallazgos estuvieron repartidos entre flujo lento coronario, ectasia coronaria, puente intramiocárdico, Takotsubo, origen anómalo y completa normalidad estructural coronaria. Cabe anotar que tres pacientes reportaron consumo de sustancias psicoactivas (pasta a base de coca).

El compromiso de un solo vaso fue lo más frecuente en esta población, y de ellos, el $89.7 \%$ debutaron con Killip-Kimball I, el 4\% con Killip-Kimball II, el 0\% con Killip-Kimball III y el $6.3 \%$ con Killip-Kimball IV.

Para el análisis bivariado, debido a la muestra pequeña, solo se obtuvieron diferencias estadísticamente significativas en pocas variables; entre ellas se encontró que, en cuanto al uso de terapia vasoactiva, de los 18 pacientes (14\%) que la requirieron, $15(83 \%)$ tenían trastornos segmentarios de la contractilidad en la ecocardiografía basal, con un valor de $p=0.021$.

\section{Discusión}

Muchos de los hallazgos obtenidos pudieran cambiar o reorientar la manera en que se aborda en el servicio de urgencias a los pacientes menores de 50 años con enfermedad cardiovascular; esta existe en pacientes jóvenes y es uno de los primeros paradigmas que se debe cambiar. Llamó la atención encontrar que el IAM no ST, con el $43 \%$, fue el SCA más prevalente. Aunque estos hallazgos concuerdan con los del estudio de
Tabla 2. Análisis estadístico de los paraclínicos evaluados

\begin{tabular}{|l|c|c|}
\hline Paraclínicos & Promedio & Mínimo-máximo \\
\hline Glucemia al ingreso & $118 \mathrm{mg} / \mathrm{dl}$ & $73-350 \mathrm{mg} / \mathrm{dl}$ \\
\hline HbA1c & $5.97 \%$ & $3.9-13.6 \%$ \\
\hline LDL basal & $109 \mathrm{mg} / \mathrm{dl}$ & $30-259 \mathrm{mg} / \mathrm{dl}$ \\
\hline HDL basal & $35 \mathrm{mg} / \mathrm{dl}$ & $21-72 \mathrm{mg} / \mathrm{dl}$ \\
\hline Triglicéridos basales & $217 \mathrm{mg} / \mathrm{dl}$ & $51-809 \mathrm{mg} / \mathrm{dl}$ \\
\hline Colesterol no HDL & $146 \mathrm{mg} / \mathrm{dl}$ & $61-309 \mathrm{mg} / \mathrm{dl}$ \\
\hline Creatinina & $1.0 \mathrm{mg} / \mathrm{dl}$ & $0.5-8.1 \mathrm{mg} / \mathrm{dl}$ \\
\hline
\end{tabular}

HbA1c: hemoglobina glucosilada; HDL: lipoproteínas de alta densidad; LDL: lipoproteínas de baja densidad.

McManus et al. ${ }^{24}$, contrastan con los del resto de los estudios, en los cuales el IAM con elevación del segmento ST era la forma de presentación más frecuente en población joven, llegando incluso a ser del $95 \%$ en uno de los reportes ${ }^{25-29}$.

Otro de los principales puntos a resaltar tiene que ver con el uso de las diferentes escalas de valoración de riesgo y pronóstico utilizadas (Killip-Kimball, TIMl y GRACE), las cuales se correlacionaron adecuadamente con el buen pronóstico intrahospitalario y mortalidad de cero; sin embargo, no se pudo establecer si se correlacionaron de igual manera en los pacientes que se estudiaron de forma ambulatoria, dado que no se contó con los datos. Debido a que se halló una alta prevalencia de enfermedad coronaria grave y que, incluso, algunos pacientes desarrollaron complicaciones hemodinámicas a pesar de haber recibido tratamiento intrahospitalario oportuno, se quiere destacar la importancia de que la decisión de realizar el estudio ambulatorio o intrahospitalario y la modalidad de estratificación coronaria (invasiva o no invasiva) no debe basarse únicamente en las escalas de riesgo.

Acerca de los factores de riesgo, de modo similar a los resultados obtenidos en el estudio conducido en Bucaramanga por Higuera et al. ${ }^{20}$, el IMC $\geq 25$ fue la principal comorbilidad encontrada, con un $71 \%$, seguido por otros factores de riesgo tradicionales, como la hipertensión arterial y la dislipidemia. Esta última tuvo, de igual manera, un hallazgo particular, pues la hipertrigliceridemia acompañada de un HDL bajo fue la alteración más frecuente hallada en el perfil lipídico, con un promedio de triglicéridos de $216 \mathrm{mg} / \mathrm{dl}$, e incluso se encontraron pacientes con triglicéridos de $809 \mathrm{mg} / \mathrm{dl}$ y 


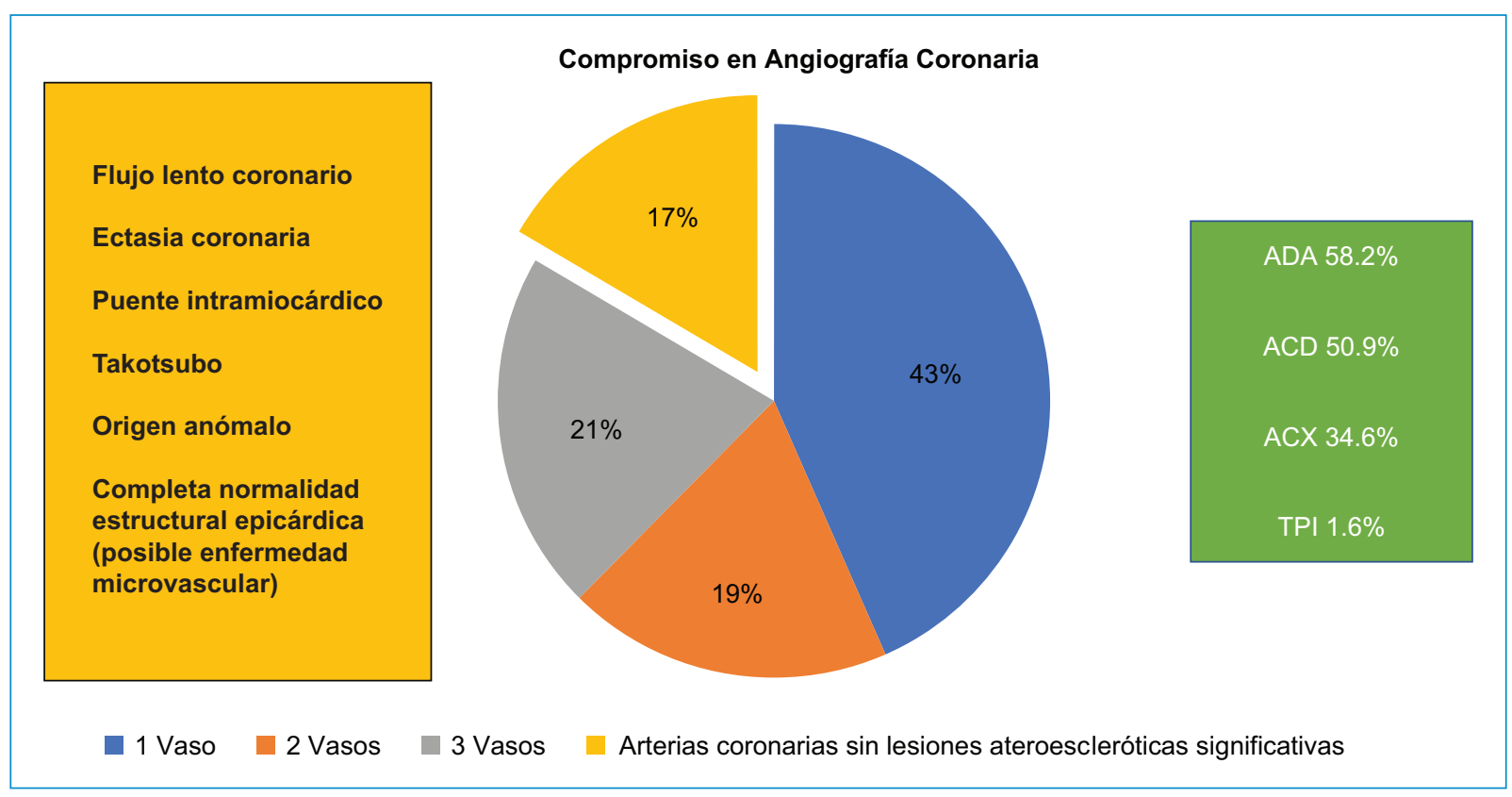

Figura 2. Hallazgos en la angiografía coronaria, tipo de lesiones y distribución de compromiso en cada arteria coronaria. ACD: arteria coronaria derecha; ACX: arteria coronaria circunfleja; ADA: arteria descendente anterior; TPI: tronco principal izquierdo.

HDL promedio de $35.5 \mathrm{mg} / \mathrm{dl}$. Estas alteraciones son comunes en los pacientes con síndrome metabólico y resistencia a la insulina, de modo que sería interesante conocer el perímetro abdominal de esta población, ya que la adiposidad visceral se ha asociado en estudios previos con el desarrollo de enfermedad cardiovascular ${ }^{30}$.

Mucho menos frecuentes fueron la diabetes mellitus, la enfermedad renal crónica y el tabaquismo o el consumo de sustancias psicoactivas; de este último contrastan los resultados con los de otros estudios en los que hacían parte de uno de los factores más prevalentes en población joven ${ }^{31}$. Como mensaje para los lectores, la ausencia de factores de riesgo tradicionales no excluye la presencia de enfermedad coronaria y, de igual manera, se debe realizar una búsqueda activa de factores de riesgo modificables en los pacientes jóvenes para realizar prevención primaria, y así mismo en aquellos que se presenten con angina de pecho y $\mathrm{SCA}^{32}$.

La distribución de las lesiones coronarias da a entender la magnitud de esta enfermedad incluso en pacientes jóvenes. La arteria descendente anterior fue la coronaria comprometida con mayor frecuencia ( $58 \%$ de los casos); el $21 \%$ tenían enfermedad coronaria grave multivaso ( $\geq 3$ vasos). En la figura 2 se muestra que en el $16.5 \%$ de los pacientes no se documentaron lesiones ateroscleróticas, con lo cual se abre la posibilidad de un grupo importante de enfermedades por estudiar y diagnosticar, diferentes al IAM tipo 1, e incluso vale la pena enfocarse en el subgrupo en el que no se documentaron lesiones en arterias epicárdicas y que tenían biomarcadores cardíacos elevados por encima del percentil 99 o una prueba de inducción de isquemia positiva, lo cual los ubica dentro del diagnóstico de IAM sin lesiones coronarias obstructivas (MINOCA, myocardial infarction with non-obstructive coronary arteries) o isquemia sin lesiones coronarias obstructivas (INOCA, ischemia with non-obstructive coronary arteries). En algunos de ellos se realizó resonancia cardíaca, que no documentó alteraciones que explicaran el cuadro clínico actual. Así mismo, 19 pacientes con cuadro clínico sugestivo de angina inestable o cambios electrocardiográficos sugestivos de isquemia fueron llevados a coronariografía, sin que se evidenciaran lesiones en arterias epicárdicas. En estos pacientes sería interesante la realización de una evaluación hemodinámica funcional para descartar vasoespasmo coronario o enfermedad microvascular, concepto que involucra la disfunción microvascular, tanto funcional como estructural, a través de diferentes índices (reserva de flujo coronario disminuida, aumento del índice de resistencia microvascular o, incluso, vasoespasmo microvascular) ${ }^{33-35}$; hallazgos que, según el estudio CORMICA, pudieran ayudar a definir el 
tratamiento farmacológico y con esto mejorar los síntomas de este tipo de pacientes ${ }^{36,37}$. A la fecha, este tipo de evaluación no se hace de forma sistemática en Colombia, pero sería un nicho interesante para considerar.

\section{Limitaciones y fortalezas}

La principal limitación está dada por ser un estudio retrospectivo, lo cual hace que solo se puedan hacer observaciones sobre la información encontrada en las historias clínicas (que en algunos casos puede ser insuficiente). De igual forma, la muestra del estudio es pequeña, ya que se recolectaron datos de un solo centro durante 3.5 años. Al compararlo con los otros estudios nacionales y los realizados en Centroamérica y América del Sur, se tiene una muestra muy similar.

\section{Conclusiones}

En este estudio, los pacientes presentaron una mezcla de factores de riesgo tradicionales y no tradicionales, pero más importante aún, se demostró que la ausencia de estos no disminuye la posibilidad de tener la enfermedad. Entre los factores de riesgo de enfermedad coronaria, en esta población predominaron el sobrepeso y la obesidad, así como la hipertrigliceridemia con HDL bajo. Adicionalmente, a pesar de que las diferentes escalas de riesgo puntúen un bajo riesgo, el criterio clínico debe primar en la decisión de realizar los estudios de forma intrahospitalaria o ambulatoria, y para definir la metodología de estratificación coronaria (invasiva o no invasiva). Es importante tener presente la posibilidad de realizar una evaluación funcional y estructural de la microcirculación, así como las pruebas de provocación de espasmo coronario en pacientes con un cuadro clínico compatible.

Los pacientes con angina de pecho, cambios en el electrocardiograma y pruebas no invasivas de inducción de isquemia positivas, en quienes la coronariografía no detecta enfermedad cardiovascular, no deberían ser considerados falsos positivos. La disfunción coronaria microvascular puede ser una etiología importante a tener en cuenta.

\section{Financiamiento}

Esta investigación no ha recibido ninguna beca específica de agencias de los sectores público, comercial o sin ánimo de lucro.

\section{Conflicto de intereses}

Los autores declaran no tener conflictos de intereses.

\section{Responsabilidades éticas}

Protección de personas y animales. Los autores declaran que para esta investigación no se han realizado experimentos en seres humanos ni en animales.

Confidencialidad de los datos. Los autores declaran que en este artículo no aparecen datos de pacientes.

Derecho a la privacidad y consentimiento informado. Los autores declaran que en este artículo no aparecen datos de pacientes.

\section{Bibliografía}

1. Ferreira-González I. Epidemiología de la enfermedad coronaria. Rev Esp Cardiol. 2014;67:139-44.

2. Ibanez B, James S, Agewall S, Antunes MJ, Bucciarelli-Ducci C, Bueno $\mathrm{H}$, et al. 2017 ESC Guidelines for the management of acute myocardial infarction in patients presenting with ST-segment elevation. Eur Heart J. 2018;39:119-77.

3. Thygesen K, Alpert JS, Jaffe AS, Chairman BR, Bax JJ, Morrow DA, et al. Fourth universal definition of myocardial infarction. J Am Coll Cardiol. 2018;72:2231-64.

4. Khera S, Kolte D, Gupta T, Subramanian KS, Khanna N, Aronow WS, et al. Temporal trends and sex differences in revascularization and outcomes of ST-segment elevation myocardial infarction in younger adults in the United States. J Am Coll Cardiol. 2015;66:1961-72.

5. Mirza AJ, Taha AY, Khdhir BR. Risk factors for acute coronary syndrome in patients below the age of 40 years. Egypt Heart J. 2018;70:233-5.

6. Chan C-M, Chen W-L, Kuo H-Y, Huang C-C, Shen Y-S, Choy C-S, et al. Circadian variation of acute myocardial infarction in young people. Am J Emerg Med. 2012;30:1461-5.

7. Awad-Elkarim AA. A prospective study of long term prognosis in young myocardial infarction survivors: the prognostic value of angiography and exercise testing. Heart. 2003;89:843-7.

8. Morillas P, Bertomeu V, Pabón P, Ancillo P, Bermejo J, Fernández C, et al. Characteristics and outcome of acute myocardial infarction in young patients. Cardiology. 2007; 107:217-25.

9. Tungsubutra W, Tresukosol D, Buddhari W, Boonsom W, Sanguanwang S, Srichaiveth B, et al. Acute coronary syndrome in young adults: the Thai ACS Registry. J Med Assoc Thail Chotmaihet Thangphaet. 2007;90 (Suppl 1):81-90.

10. Malmberg K, Båvenholm $P$, Hamsten A. Clinical and biochemical factors associated with prognosis after myocardial infarction at a young age. J Am Coll Cardiol. 1994;24:592-9.

11. Avezum A, Makdisse M, Spencer F, Gore JM, Fox KAA, Montalescot G, et al. Impact of age on management and outcome of acute coronary syndrome: observations from the global registry of acute coronary events (GRACE). Am Heart J. 2005;149:67-73.

12. Shah N, Kelly A-M, Cox N, Wong C, Soon K. Myocardial infarction in the "young": risk factors, presentation, management and prognosis. Heart Lung Circ. 2016;25:955-60.

13. Shukla AN, Jayaram AA, Doshi D, Patel P, Shah K, Shinde A, et al. The young myocardial infarction study of the Western Indians. Glob Heart. 2019;14:27-33.

14. Aggarwal A, Aggarwal S, Goel A, Sharma V, Dwivedi S. A retrospective case-control study of modifiable risk factors and cutaneous markers in Indian patients with young coronary artery disease. JRSM Cardiovasc Dis. 2012;1:1-8.

15. Kianoosh Hosseini S, Soleimani A, Ali Karimi A, Sadeghian S, Darabian S, Hesameddin Abbasi S, et al. Clinical features, management and in-hospital outcome of ST elevation myocardial infarction (STEMI) in young adults under 40 years of age. Monaldi Arch Chest Dis. 2009;72:71-6.

16. Kannel WB, Abbott RD. Incidence and prognosis of unrecognized myocardial infarction: an update on the Framingham Study. N Engl J Med. 1984;311:1144-7. 
Rev Colomb Cardiol. 2022;29(1)

17. Osula S. Acute myocardial infarction in young adults: causes and management. Postgrad Med J. 2002;78:27-30.

18. Chaudhary P, Agarwal N, Kulshrestha M, Kumaran A, Chaudhary S Gupta S. Assessment of myocardial infarction in young patients. IJCMR. 2015;3:4.

19. Lee $\mathrm{SH}, \mathrm{Kim} \mathrm{JH}$, Jeong MH, Park H, Jeong YA, Ahn Y, et al. Clinical characteristics and outcomes of acute ST-segment elevation myocardial infarction in younger Korean adults. Korean Circ J. 2015;45:275

20. Higuera SA, Hernández-Delgado LM, Vesga BE. Síndrome coronario agudo en adultos jóvenes llevados a arteriografía coronaria. Rev Colomb Cardiol. 2020;27:77-83.

21. Wiesbauer F, Blessberger H, Azar D, Goliasch G, Wagner O, Gerhold L, et al. Familial-combined hyperlipidaemia in very young myocardial infarction survivors ( $\leq 40$ years of age). Eur Heart J. 2009;30:1073-9.

22. Roffi $M$, Patrono $C$, Collet JP, Mueller C, Valgimigli $M$, Andreotti F, et al. 2015 ESC Guidelines for the management of acute coronary syndromes in patients presenting without persistent ST-segment elevation: Task Force for the Management of Acute Coronary Syndromes in Patients Presenting without Persistent ST-Segment Elevation of the European Society of Cardiology (ESC). Eur Heart J. 2016;37:267-315.

23. Lang RM, Badano LP, Mor-Avi V, Afilalo J, Armstrong A, Ernande L, et al. Recommendations for cardiac chamber quantification by echocardiography in adults: an update from the American Society of Echocardiography and the European Association of Cardiovascular Imaging. J Am Soc Echocardiogr. 2015;28:1-39.e14.

24. McManus DD, Piacentine SM, Lessard D, Gore JM, Yarzebski J, Spencer FA, et al. Thirty-year (1975 to 2005) trends in the incidence rates, clinical features, treatment practices, and short-term outcomes of patients $<55$ years of age hospitalized with an initial acute myocardial infarction. Am J Cardiol. 2011;108:477-82.

25. Lei L, Bin Z. Risk factor differences in acute myocardial infarction between young and older people: a systematic review and meta-analysis. Int J Cardiovasc Sci. 2019;32:163-76.

26. Malik FTN, Kalimuddin MD, Ahmed N, Badiuzzzaman M, Ahmed MN Dutta $A$, et al. AMI in very young (aged $\leq 35$ years) Bangladeshi patients: risk factors and coronary angiographic profile. Clin Trials Regul Sci Cardiol. 2016;13:1-5.
27. Rubido YE, Sáez GC, Valdivié IQ, Rodríguez RMP, López DG. Infarto agudo de miocardio en pacientes menores de 50 años en el Hospital Arnaldo Milián Castro. Revista Cubana de Cardiología y Cirugía Cardiovascular. 2018;24:15

28. Valdés Martín A, Martínez Benítez P, Chipi Rodríguez Y, Reyes Navia G, Echevarría Sifontes L, Rivas-Estany E. Caracterización del síndrome coronario agudo en adultos menores de 45 años de una institución especializada en la Habana, Cuba, entre 2013 y 2014. Médicas UIS. 2015;28:281-90.

29. Bhardwaj R, Kandoria A, Sharma R. Myocardial infarction in young adults risk factors and pattern of coronary artery involvement. Niger Med J. 2014;55:44.

30. Di Angelantonio E, Bhupathiraju SN, Wormser D, Gao P, Kaptoge S, de González $A B$, et al. Body-mass index and all-cause mortality: individual-participant-data meta-analysis of 239 prospective studies in four continents. Lancet. 2016;388:776-86.

31. Méndez M, Martínez G, Veas N, Pérez O, Lindefjeld D, Winter JL, et al. Infarto agudo al miocardio en pacientes menores de 40 años: características clínicas, angiográficas y alternativas terapéuticas. Rev Chil Cardiol. 2013;32:21-7.

32. Yusuf S, Hawken S, Ônpuu S, Dans T, Avezum A, Lanas F, et al. Effect of potentially modifiable risk factors associated with myocardial infarction in 52 countries (the INTERHEART study): case-control study. Lancet. 2004;364:937-52.

33. Ong P, Camici PG, Beltrame JF, Crea F, Shimokawa H, Sechtem U, et al. International standardization of diagnostic criteria for microvascular angina. Int J Cardiol. 2018;250:16-20.

34. Padro T, Manfrini O, Bugiardini R, Canty J, Cenko E, De Luca G, et al. ESC Working Group on Coronary Pathophysiology and Microcirculation position paper on 'coronary microvascular dysfunction in cardiovascular disease'. Cardiovasc Res. 2020;116:741-55

35. Taqueti VR, Di Carli MF. Coronary microvascular disease pathogenic mechanisms and therapeutic options. J Am Coll Cardiol. 2018;72:2625-41.

36. Ford TJ, Stanley B, Good R, Rocchiccioli P, McEntegart M, Watkins S, et al. Stratified medical therapy using invasive coronary function testing in angina. J Am Coll Cardiol. 2018;72:2841-55.

37. Ford TJ, Stanley B, Sidik N, Good R, Rocchiccioli P, McEntegart M, et al. 1-Year outcomes of angina management guided by invasive coronary function testing (CorMicA). JACC Cardiovasc Interv. 2020;13:33-45. 